\title{
Rhithrogena ourika n. sp., Ephéméroptère nouveau du Haut Atlas marocain (Heptageniidae)
}

\author{
A.G.B. Thomas I \\ A. Mohati ${ }^{2}$
}

Mots clés : Ephemeroptera, Heptageniidae, Nouvelle espèce, Maroc.

L'imago ơ de Rhithrogena ourika n. sp., récoltée dans le Haut Atlas (Maroc) est décrite et figurée. Il s'agit de la première espèce de Rhithrogena identifiée en Afrique du Nord.

\section{Rhithrogena ourika n. sp., a new mayfly from the moroccan Haut Atlas (Ephemeroptera, Heptagenlidae)}

Keywords : Ephemeroptera, Heptageniidae, New species, Morocco.

The o imagine of Rhithrogena ourika n. sp., found in the Haut Atlas (Morocco) is described and illustrated. This is the first Rhithrogena species to be named from North Africa.

La première citation du genre Rhithrogena en Af rique du Nord est déjà fort ancienne (Eaton, 1899). Elle concerne une capture, effectuée à Biskra (Algérie) dont le spécimen n'a pas été identifié spécifiquement. Les citations ultérieures sont aussi restées au niveau générique. Lestage en particulier (1925), a seulement repris Eaton (t.c.). Dakki (1979 et communication verbale récente) indique la présence d'une espèce de Rhithrogena dans le cours moyen et surtout inférieur de l'oued Sebou (Moyen Atlas marocain). Cette espèce de basse altitude, thermophile, colonise des rivières assez larges et à pente modérée. Dakki (t.c.) pense qu'il s'agit probablement de l'espèce rencontrée par Eaton en Algérie.

L'étude de la faune benthique de plusieurs réseaux hydrographiques des environs de Marrakech, sous la direction de M.J. Giudicelli (Marseille)

1. Laboratoire d'Hydrobiologie, UA 695 du C.N.R.S., Université Paul Sabatier, 118, route de Narbonne, 31062 Toulouse Cedex, France.

2. Département des Sciences de la Vie, Faculté des Sciences, Universite Cadi Ayyad, Boulevard de Safi, B.P. S 15, Marrakech, Maroc.

Ce travail a été réalisé dans le cadre de l'action de Recherche Intégrée franco-marocaine $n^{\circ} 24$ : * Ecologie des milieux aquatiques continentaux des pays méditerraneens. et de M.J.C. Pihan (Metz) - effectuée sur une très grande amplitude altitudinale - a montré que les larves du genre Rhithrogena récoltées dans cette région appartiennent au moins à deux espèces distinctes. En particulier, des cours d'eau du bassin versant de l'oued Ourika (massif montagneux de l'Oukaimeden) abritent, à haute altitude, une espece très caractéristique du rhithron, qui est nouvelle et dont voici la description.

\section{Rhithrogena ourika n. sp.}

Imago $\sigma$ (conservation en alcool à $70^{\circ}$ ).

Diagnose sommaire: espece de taille moyenne: coloration d'ensemble brun moyen, ailes claires sauf le ptérostigma; pénis simple, dépourvu de fortes denticulations.

Tête.

Face gris brunâtre ; vertex brun foncé. Ocelles gris clair, les mamelons ocellaires cerclés de brun noirâtre. Yeux rosâtres devenant progressivement bleu noirâtre vers le bord externe. Antennes brunes, le funicule plus foncé et plus grisâtre.

Thorax.

Prothorax. Tergite brun foncé de même que la région centrale, proéminente, du sternite. Pattes 1 

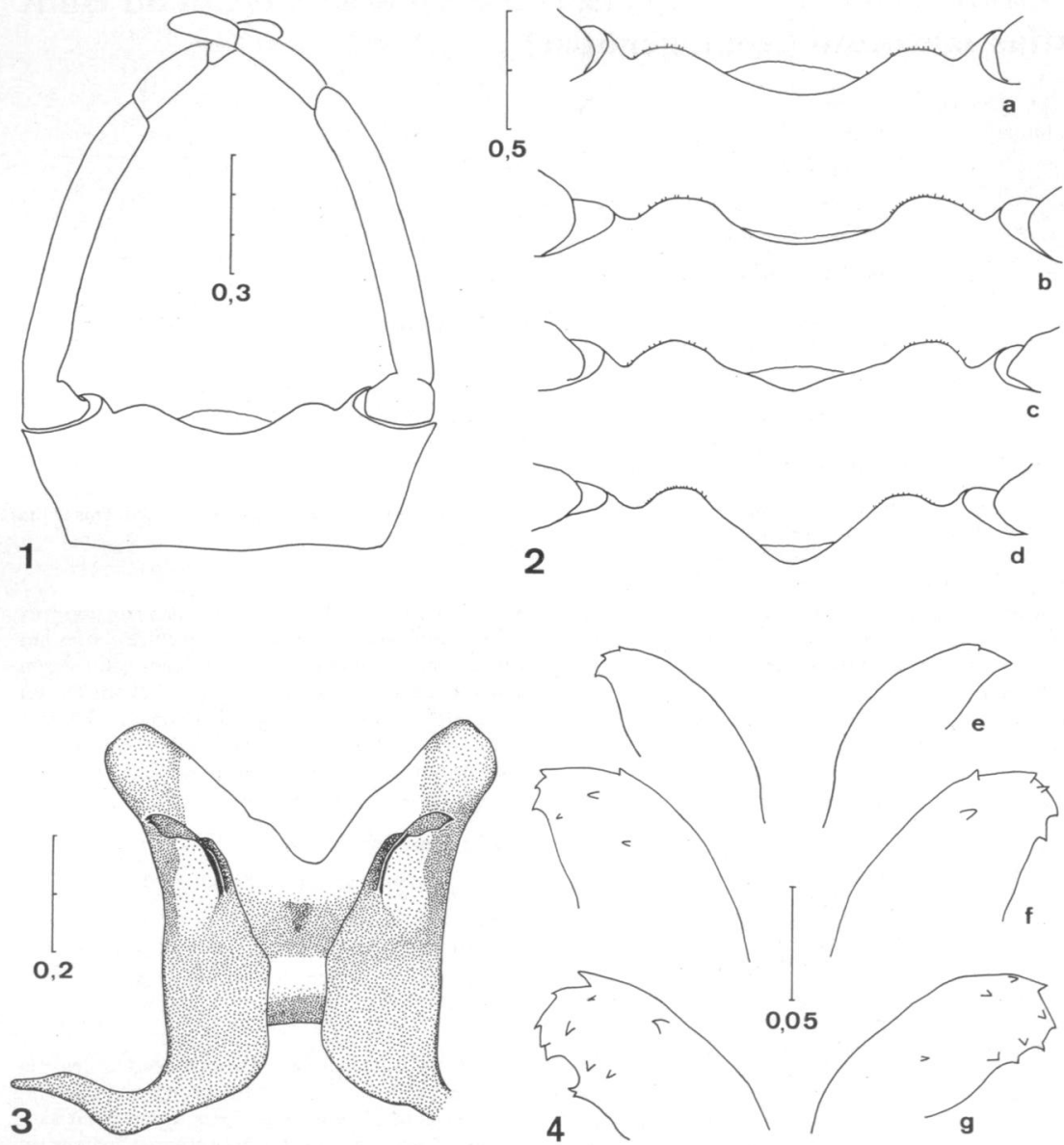

Fig. 1 à 4 : imago o de Rhithrogena ourika n. sp. Echelle en mm. 1 : styligère et styles en vue ventrale. 2 a à $\mathrm{d}$ : variations du bord postérieur du styligère sur 4 individus. 3 : pénis en vue ventrale. $4 \mathrm{e}$ à $\mathrm{g}$ : titillateurs chez 3 individus. 


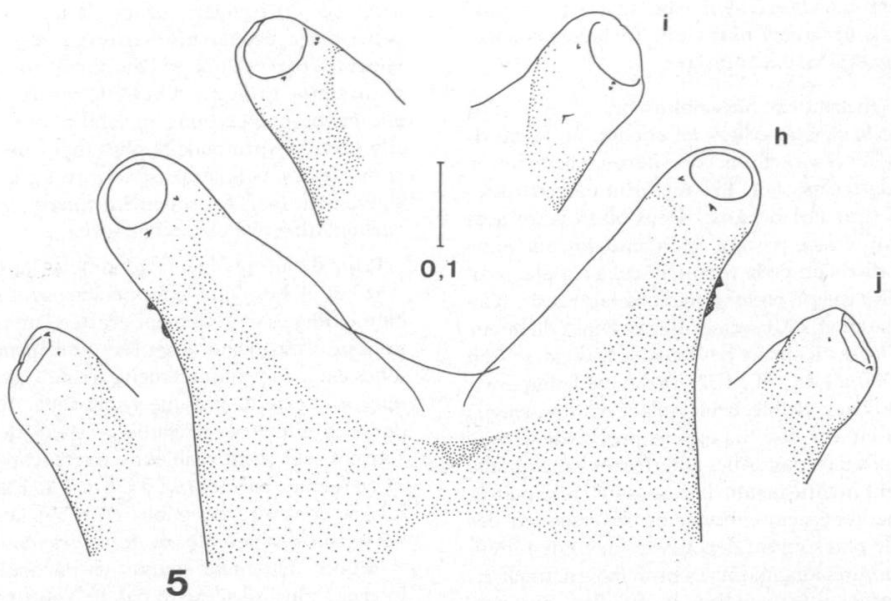

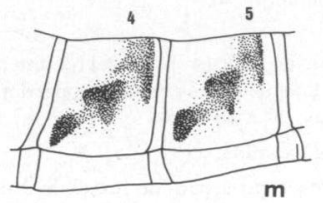

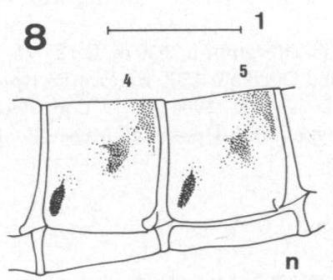

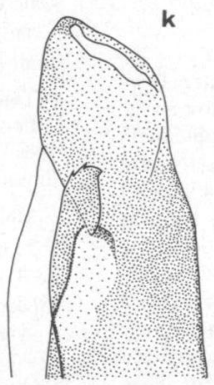
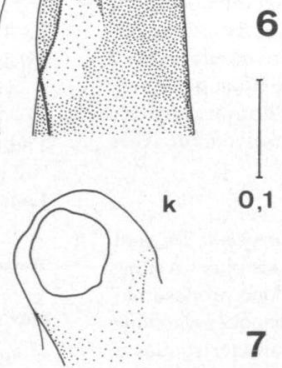
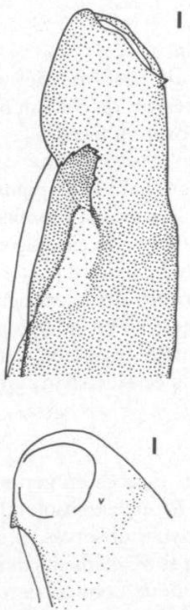

Fig. 5 à 8 : imago $\sigma$ de Rhithrogena ourika n. sp. Echelle en mm. 5 h : lobes péniens en vue dorsale (i et $\mathrm{j}$ : variations de la région apicale sur deux autres individus). $6 \mathrm{k}$ et $1:$ pénis en vue latérale gauche, sans épine latéro-dorsale (k) et avec la plus grande épine de la série type (1). $7 \mathrm{k}$ et $\mathrm{I}$ : les mêmes lobes péniens en vue dorso-apicale. 8 : coloration latérale des $4^{\circ}$ et $5^{\circ}$ tergites abdominaux chez un exemplaire très pigmenté (m) et chez un exemplaire clair (n). N.b. : en raison de la taille réduite des denticulations, la pigmentation n'a pas été représentée sur l'apex des lobes péniens. (fig. 5 et 7 ). 
entièrement brun foncé, sauf le tiers ou le quart basal des témurs, nettement plus clair. Tache médiofémorale en général visible, noirâtre.

Méso et métathorax. Mesonotum brun moyen, plus foncé dans la région médiane antérieure, sur les bords et surtout sur le scutellum. Mésosternum bran foncé, métasternum plus clair. Pleures brun moyen, membranes pleurales blanchâtres. Deux ou trois traînées de pigment violacé partent de la base de l'aile antérieure en direction de la première coxa : la plus visible se trouve dans le prolongement du bord costal. Pattes 2 et 3 jaune grisâtre, rembrunies à l'apex du fémur et sur les tarses. Rapport des longueurs du tarse 1 au tibia $1: 1,20$ à 1,$45 ; \bar{m}: 1,32$. Tache médiofémorale étroite mais bien visible, brun violacé intense, longue comme la largeur du tibia et plus proche de la base que de l'apex du fémur. Ailes antérieures à membrane entièrement hyaline depuis la base, sauf l'aire ptérostigmatique, fortement enfumée et traversée par des nervures le plus souvent dépourvues de toute ramification. Nervures longitudinales brun moyen, transverses plus larges et brun violacé jusqu'à R2. Ailes postérieures hyalines à nervation claire, tout au plus brun grisâtre.

\section{Abdomen.}

Couleur de base brun terne à gris jaunâtre. Dans le coin antérieur de chacun des tergites 2 (ou 3) à 8 . une tache brun violacé foncé, ovale, bien individualisée (fig. $8 \mathrm{~m}$ ). Sur la plupart des exemplaires, il existe en outre une trainée de pigment latérale oblique rattachée ou non à la première tache-qui traverse chaque demi-tergite en diagonale, en direction de la partie médiane du bord postérieur (fig. $8 \mathrm{~m}$ ). Cette dernière coloration peut cependant être atténuée (fig. 8 n). Stemites brun teme uniforme, le $9^{\circ}$ jaunâtre avec un bord antérieur violacé. Cerques brun très foncé à la base, s'éclaircissant progressivement vers l'extrémité.

\section{Genitalia.}

Parmi les espèces du genre Rhithrogena, les genitalia $\sigma$ de $R h$ ourika sont, de loin, les plus variables que nous ayons observés. Il sera donc proposé plusieurs figures des éléments morphologiques classiquement considérés comme les plus caractéristiques et les plus stables.

Styligère (fig. 1), brun jaunâtre dans la région médiane, plus foncé près du bord postérieur et latéralement, sous l'insertion des styles. Protubérances médianes du styligère planes, de taille relativement petite, assez régulièrement arrondies en général et nettement séparées l'une de l'autre par une large échancrure concave (fig. 2 a et b). Cette échancrure présente elle même une certaine variabilité morphologique : elle peut être profonde et plus anguleuse (fig. $2 \mathrm{~d}: 2$ ơ sur les 24 examinés). Styles brun grisâtre moyen, légèrement mais brusquement rétrécis au-dessus de l'articulation avec le styligère (fig. 1).

Pénis de morphologie très simple dans l'ensemble (fig. 3 et 5). Les deux lobes péniens sont assez longs, subrectilignes et nettement écartés l'un de l'autre presque orthogonaux avant l'accouplement. L'apex des lobes est assez régulièrement arrondi, avec un profil interne et externe presque symétrique. Epines latérodorsales et internes irrégulières, très réduites, le plus souvent seulement visibles au microscope (fig. 5, 6, et 7), ou même absentes (fig. 5 j. 6 et 7 k). Elles sont parfois, au contraire, dédoublées (fig. 5 i). Orifice pénien dorso-apical, à peu près ovale, de grandes dimensions et à bord nettement épaissi, en particulier du côté interne : ainsi, il n'existe pas de gouttière pénienne dorso-inteme proprement dite mais seulement une zone membraneuse n'atteignant pas l'apex.

Titillateurs plutôt larges, à nombre de denticulations variable (fig. 4).

Taille.

Longueur de l'aile $\left(\sigma^{\circ}\right): 9,6$ à $11,2 \mathrm{~mm}$ (moyenne : $10,3 \mathrm{~mm}$ ). Longueur des cerques proportionnellement importante : 20 à $28 \mathrm{~mm}$.

\section{Matériel examiné.}

24 imagos $\sigma$ provenant du réseau hydrographique de l'oued Ourika (massif montagneux de l'Oukaimeden) :

- l'assif Tiferguine à $2620 \mathrm{~m}$, le 12 - VI - $1984: 3 \sigma$.

- l'oued Ourika à $1500 \mathrm{~m}$ (localité typique : Setti Fadma), le 12 - VI - $1984: 21 \sigma$ dont lholotype.

2 paratypes sont déposés au Musée Zoologique de Lausanne.

\section{Travaux cités}

Dakki (M.). 1979. - Recherches hydrobiologiques sur un cours d'eau du Moyen Atlas (Maroc). Thèse de spécialité, Univ. Aix-Marseille III. 126 p. +7 pl.

Eaton (AE.). 1899. - List of Ephemeridae hitherto observed in Alge. ria with localities. Entomologist's mon. Mag., 35 : $4-5$.

Lestage (J.A.). 1925. - Ephémeroptères, Plécoptères et Trichoptères recueillis en Algérie par M.H. Gauthier et liste des especes connues actuellement de l'Afrique du Nord. Bull Soc. Hist. nat. Afr. N., $16: 8-18$. 